\title{
Determining if Double Gene Knockdown of P65 and PDK2 Increases Cytotoxicity and Radiation Sensitivity of Pancreatic Cancer Cells
}

\author{
Madison Tenbarge ${ }^{1}$, Devyn Townsend ${ }^{2}$, Ryan Erdwin², Helen Chin-Sinex², Marc \\ Mendonca ${ }^{2,3}$ \\ ${ }^{1}$ Indiana University School of Medicine, ${ }^{2}$ Department of Radiation Oncology, \\ ${ }^{3}$ Department of Medical and Molecular Genetics \\ Indiana University School of Medicine, Indianapolis, IN 46202
}

Background: Pancreatic ductal adenocarcinoma (PDA) is currently the $4^{\text {th }}$ leading cause of cancer death in the US because of late detection and resistance to chemotherapy and radiation. This tumor resistance is partially due to high activity of the transcription factor, NF- $\mathrm{BB}$ which promotes cell survival and pyruvate dehydrogenase kinase 2 (PDK2) which regulates aerobic glycolysis (Warburg Metabolism), cell proliferation, and inhibition of apoptosis. We have shown that simultaneous treatment with the chemical NF-kB inhibitor DMAPT and the Warburg inhibitor DCA is cytotoxic and enhances radiation-induced cell death in human PDA cells.

Experimental Design: We hypothesize that double gene knockdown of the P65 subunit of NF-kB and PDK2 by siRNA will lead to increased cytotoxicity and radiation sensitivity. The PDA cell line (Mia PaCa-2) was utilized and was transfected with appropriate siRNAs. The cells were plated and followed by irradiation with either 0,2 , 4, or 6 Gy of 160 kVp X-rays.

Results: Analysis of Western blots shows the successful silencing of P65, but the knockdown of PDK2 are pending. The differences in plating efficiency were not found to be statistically significant between the treatment groups. The differences in survival fraction were not found to be statistically significant at 2, 4, or $6 \mathrm{~Gy}$, but there is a trend for enhancement at $6 \mathrm{~Gy}$.

Conclusion: Preliminary data indicate there was no increase in cytotoxicity or radiation-induced cell killing after treatment with SiP65, SiPDK2, or both. However, to date we have no evidence that siPDK2 is working and further experiments are underway. 